\title{
Robotic Foregut Surgery: One Surgeon's Experience with Nissen Fundoplication, Esophagomyotomy, and Hiatal Hernia Repair
}

\author{
Franziska Huettner ${ }^{1}$, Robin A. Alley ${ }^{2}$, Jamie L. Doubet ${ }^{2}$, Michael J. Ryan ${ }^{3}$, \\ Danuta I. Dynda ${ }^{2}$, David L. Crawford ${ }^{2 *}$ \\ ${ }^{1}$ Division of Plastic Surgery, Department of Surgery, University of Alabama at Birmingham, \\ Birmingham, UK \\ ${ }^{2}$ Division of Minimally Invasive Surgery, Department of Surgery, College of Medicine, University of Illinois at Peoria, \\ Illinois Medical Center, Peoria, USA \\ ${ }^{3}$ Department of Radiology, University of Michigan Hospital, Ann Arbor, USA \\ Email: *lapsurg@comcast.net
}

Received July 24, 2011; revised October 25, 2011; accepted November 22, 2011

\begin{abstract}
Purpose: The purpose of the study was to review a single surgeon, seven-year experience, using the Da Vinci Telerobotic system performing 124 foregut operations. Methods: Data review from 2002-2009 including 71 Nissen fundoplications, 26 esophagomyotomies, and 27 hiatal hernia repairs was performed. Parameters collected included gender, age, body mass index (BMI), estimated blood loss (EBL), port set up time (PST), robot operating time (ROT), total case time (TCT), length of stay (LOS), complications, conversions, and resident involvement. Statistical analysis was conducted. Results: 124 foregut operations: 45 males, 79 females, mean age of $54.8 \pm 16.7$ (18 - 85) years, mean TCT $174.4 \pm 45.0$ (102 - 321) min. Nissen fundoplication: mean BMI of $30.8 \pm 3.9(22.4-46.8) \mathrm{kg} / \mathrm{m}^{2}$, EBL $30.2 \pm 21.8(5-$ 100) ml, PST $32.3 \pm 9$ (14 - 63) min, ROT $111.4 \pm 37.3$ (51 - 229) min, TCT $175.0 \pm 46.4$ (102 - 321) min, median LOS 1 (0 - 9) day, complication rate 7.0\% (5/71), conversion rate 5.6\%, resident involvement $69.0 \%$ (49/71). Esophagomyotomy: mean BMI of $26.5 \pm 6.1(15.4-36.6) \mathrm{kg} / \mathrm{m}^{2}$, EBL $39.1 \pm 41.7$ (10 - 200) ml, PST $28.0 \pm 8.6$ (16 - 47) min, ROT $122.9 \pm 45$ (31 - 217) min, and TCT $178.0 \pm 40.5$ (105 - 262) min, median LOS 1 (0 - 6) day, complication rate $15.4 \%(4 / 26)$, conversion rate $0 \%$, resident involvement $69.2 \%$ (18/26). Hiatal hernia repair: mean BMI of $28.4 \pm 4.2$ $(21.9$ - 36.8) kg/m², EBL $38.4 \pm 32.7$ (10 - 150) ml, PST $28.8 \pm 8.0$ (17 - 52) min, ROT $109.0 \pm 44.5$ (49 - 250) min, and TCT $169.2 \pm 46.5(102-299)$ min, median LOS 1 (1 - 14) day, complication rate 11.1\% (3/27), conversion rate $3.7 \%$, resident involvement $66.7 \%$ (18/27). Conclusion: Robotic-assisted foregut surgery is safe and effective. This series compares favorably with other robotic studies in length of hospital stay, total case time, and complication and conversion rates. Foregut surgery is an excellent robotic training ground for residents.
\end{abstract}

Keywords: Robot; Foregut; Nissen Fundoplication; Hiatal Hernia; Esophagomyotomy

\section{Introduction}

Laparoscopic surgery for procedures such as Nissen fundoplication and esophagomyotomy are commonly performed. Compared to conventional surgery, laparoscopic surgery has shown similar outcomes with decreased post-operative pain and shortened hospital stays. However, laparoscopy creates its own challenges and requires high skill levels especially in foregut surgery. Performing complex maneuvers in the confined subphrenic space with the use of long instruments, with limited degrees of freedom, through fixed insertion points can prove to be quite difficult.

\footnotetext{
${ }^{*}$ Corresponding author.
}

Robotic surgery has been shown to be a safe and effective alternative to laparoscopy with added benefits [1] The Food and Drug Administration approved the da Vinci Robotic System ${ }^{\circledR}$ Intuitive Surgical Inc. Sunnyvale, CA for intra-abdominal surgery in 2000. This system provides instruments with articulated wrists to allow for seven degrees of motion, tremor filtration, comfortable ergonomics, up to ten fold magnification, and three-dimensional imaging. These benefits allow the surgeon to perform advanced procedures with greater precision and comfort. Robotic surgery has disadvantages as well, including loss of tactile sensation, high costs, and extended operative time. The first robotic Nissen fundoplication was reported in 1999 and was among the first procedures 
to be performed robotically [2]. The first robotic esophagomyotomy was reported in 2001 [3]. However, there is still debate as to whether robotic surgery has a significant advantage over the standard laparoscopic techniques.

The objective of this study is to present data from our first 124 consecutive robotic foregut procedures, including Nissen fundoplications, esophagomyotomies, and hiatal hernia repairs and compare the results with those in the current robotic literature.

\section{Methods}

A retrospective review was performed on prospectively collected data from 124 robotic foregut procedures performed by one minimally invasive surgery fellowshiptrained surgeon (DLC) using the da Vinci robotic system ${ }^{\circledR}$ over an eight-year period (2002-2009). These cases included 71 Nissen fundoplications, 26 esophagomyotomies, and 27 hiatal hernia repairs.

\subsection{Preoperative Assessment}

Patients with symptomatic gastroesophageal reflux disease (GERD) and hiatal hernias underwent a workup that included esophagogastroduodenoscopy (EGD), 24-hour $\mathrm{pH}$ probe, and esophageal manometry with nuclear gastric emptying study being performed on selected patients. Patients with achalasia underwent a workup that included an EGD, upper GI, and esophageal manometry. All hiatal hernia patients without symptomatic GERD underwent an EGD, while a barium upper gastrointestinal series (UGI) was performed selectively. If a minimally invasive approach to surgery was feasible and the patient's chosen hospital had a robot, then a robotic approach was scheduled. Pertinent risks and benefits were discussed with every patient and informed consent was obtained prior to surgery.

\subsection{Operative Technique}

All procedures were performed using the da Vinci System $^{\circledR}$. The system consists of the surgeon's console, the surgical cart with three or four robotic arms to which the camera and instruments are attached, and the vision tower. The surgeon sits at the console and from there controls the camera and the instrument arms with two hand manipulators (masters) and foot pedals. The surgeon's movements are electronically transferred, in realtime, to the tips of the instruments.

The port size and placement were similar for all 3 procedures (Figure 1).

A footboard and thigh strap is used to secure the patient to the table. The right arm is tucked at the side to allow the liver retractor to be anchored to that side of the table.

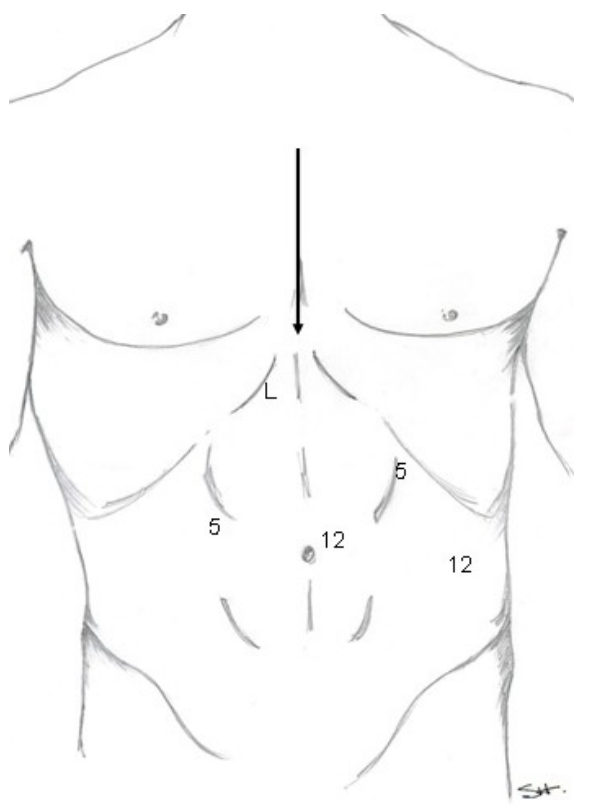

Figure 1. Trocar positioning foregut procedures. $(\mathrm{L}=$ liver retractor, $5=5 \mathrm{~mm}$ trocar site, $12=12 \mathrm{~mm}$ trocar site, Arrow indicates direction of approach for robot).

After insufflation of the abdomen to $15 \mathrm{mmHg}$, a 12 -mm port is placed $2 \mathrm{~cm}$ to the left and $3 \mathrm{~cm}$ cephalad of the umbilicus. A 30 degree laparoscope is introduced through the port and the remaining trocars are placed under direct visualization, including a 5-mm trocar placed in the left upper quadrant, 12-mm trocar in the left lateral abdominal wall, a 5-mm in the right upper quadrant, and a liver retractor is placed through a 5-mm incision in the epigastric area. The patient is then placed in steep reverse Trendelenburg position. The da Vinci is brought in over the patient's head and is docked to the ports (see arrow in Figure 1). Nissen fundoplication, esophagomyotomy, or hiatal hernia repair was then performed using established techniques.

All patients had follow up appointments at 1 week, 1 month, 6 months, and 1 year. Follow up testing was only performed in GERD and achalasia patients if they were experiencing symptoms. The hiatal hernia repair patients underwent routine annual barium upper gastrointestinal series (UGI) to evaluate their repair since many failures are symptomatically silent.

Data collected from all three groups included gender, age, body mass index (BMI), estimated blood loss (EBL), port set up time (PST), robot operating time (ROT), total case time (TCT), length of stay (LOS), complications, conversions, and resident involvement. The PST was defined as the time from the first skin incision until the surgeon sat down at the console to begin the robotic portion of the procedure. The ROT was defined as the first time the robot was used until it was disengaged from the trocars. The TCT was from first skin incision until skin 
closure. Statistical analysis was performed using the Anova test.

\section{Results}

A total of 124 robotic assisted foregut operations were performed on 45 males and 79 females. These included 71 Nissen fundoplications for GERD, 26 esophagomyotomies for achalasia, and 27 hiatal hernia repairs. The patient demographics for all three procedures are seen in Table 1.

Total times for all the foregut cases combined: PST $30.6 \pm 8.8$ (14 - 63) minutes, ROT $113.3 \pm 40.6$ (31 - 250) minutes, TCT $174.4 \pm 29.4$ (102 - 321) minutes. The mean LOS for all cases combined was 1.7 (0 - 14) days. Residents were involved in the cases $68.5 \%$ (85/124) of the time.

In the Nissen fundoplication group times were: PST $32.3 \pm 9$ (14 - 63) minutes, ROT $111.4 \pm 37.3$ (51 - 229) minutes, and TCT $175 \pm 46.4$ (102 - 321) minutes. The mean LOS was 1.7 (0 - 9) days. Residents were involved in the Nissen fundoplication cases $69.0 \%(49 / 71)$ of the time. Four cases were converted laparoscopy (5.6\%) and none to open (Table 1). The complications in the Nissen fundoplication group are seen in Table 2.

All of the Nissen fundoplications except one had a standard hiatal closure. Twenty-five cases (35.2\%) had additional procedures performed at the time of surgery (Table 3).

Times for the esophagomyotomy group were: PST $28 \pm$ 8.6 (16 - 47) minutes, ROT $122.9 \pm 45$ (31 - 217) min- utes, and TCT $178 \pm 40.5$ (105 - 262) minutes. The mean LOS was $1.2(0$ - 6) days. Residents were involved in the esophagomyotomies $69.2 \%$ (18/26) of the time. No cases were converted to laparoscopy or open approach (Table 1). There were four complications in the esophagomyotomy group (Table 2). Twenty-two cases (84.6\%) had additional procedures at the time of surgery (Table 3).

Hiatal hernia repair operative times were: PST $28.8 \pm$ 8 (17 - 52) minutes, ROT $109 \pm 44.5$ (49 - 250) minutes, and TCT $169.2 \pm 46.5$ (102 - 299) minutes. The mean LOS was 2.3 (1 - 14) days. Residents were involved in the hiatal hernia repairs $66.7 \%(18 / 27)$ of the time. One case was converted to laparoscopy and none were converted to open (Table 1). There were three complications in the hiatal hernia repair group (see Table 2). Seventeen cases (63\%) had additional procedures at the time of surgery (Table 3 ).

\section{Discussion}

\subsection{Complications}

Of the Nissen fundoplication group, one patient developed an incarcerated paraesophageal hernia. The patient presented with severe dysphagia 14 months post-operatively. Chest x-ray and barium UGI showed a large hiatal hernia. Diagnostic laparoscopy was performed revealing an incarcerated paraesophageal hernia. It was reduced and repaired then reinforced with human dermal allograft laparoscopically. Another patient had a presumed vagal nerve injury. The patient developed persistent nausea and

Table 1. Data by procedure group.

\begin{tabular}{lccc}
\hline \multicolumn{1}{c}{ Data Point } & Nissen Fundoplication & Esophagomyotomy & Hiatal Hernia Repair \\
\hline $\mathrm{n}$ & 71 & 26 & 27 \\
Male & 21 & 15 & 9 \\
Female & 50 & 11 & 18 \\
Age mean (range) years & $50.2(27-84)$ & $50.6(18-80)$ & $71.1(49-85)$ \\
BMI mean (range) kg/m² & $30.8(22.4-46.8)$ & $26.5(15.4-36.6)$ & $28.4(21.9-36.8)$ \\
PST mean (range) min & $32.3(14-63)$ & $28.0(16-47)$ & $28.8(17-52)$ \\
ROT mean (range) min & $111.4(51-229)$ & $122.9(31-217)$ & $109.0(49-250)$ \\
TCT mean (range) min & $175.0(102-321)$ & $178.0(105-262)$ & $169.2(102-299)$ \\
EBL mean (range) ml & $30.2(5-100)$ & $39.1(10-100)$ & $38.4(10-150)$ \\
LOS mean (range) days & $1.7(0-9)$ & $1.2(1-6)$ & $2.3(0-14)$ \\
Resident participation n (\%) & $49 / 71(69.0)$ & $18 / 26(69.2)$ & $18 / 27(66.7)$ \\
Conversion & & & $1(3.7)$ \\
To Laparoscopy n (\%) & $4(5.6)$ & 0 & 0 \\
To Laparotomy n (\%) & 0 & 0 & \\
\hline
\end{tabular}


Table 2. Complications.

\begin{tabular}{ccc}
\hline Nissen Fundoplication & Esophagomyotomy & Hiatal Hernia Repair \\
\hline DVT & Liver puncture & Gastroparesis \\
DVT/PE & Submucosal bleed & Wound infection \\
Vagal nerve injury & Splenic bleed & Prolonged intubation \\
Prolonged intubation & $\begin{array}{c}\text { Gastric perforation } \\
\text { during myotomy }\end{array}$ & \\
Paraesophageal hernia & & \\
\hline
\end{tabular}

Table 3. Additional procedures performed during foregut operations.

\begin{tabular}{cl}
\hline $\mathrm{n}$ & \multicolumn{1}{c}{ Nissen Fundoplication } \\
\hline 45 & Hiatal Hernia Repair (HHR) \\
14 & HHR \& Anterior Gastropexy \\
4 & HHR \& Cholecystectomy \\
3 & HHR \& Pyloroplasty \\
2 & HHR \& Collis Gastroplasty \\
1 & HHR \& Gastric Pacemaker Placement \\
1 & HHR, Collis Gastroplasty, \& Anterior Gastropexy \\
\hline 18 & \multicolumn{1}{c}{ Esophagomyotomy } \\
\hline 2 & Toupet Fundoplication \\
1 & HHR Fundoplication \\
1 & Toupet Fundoplication \& Pyloroplasty \\
\hline 14 & Anterior gastropexy \\
\hline 2 & Gastric Tube Placement \\
1 & Cholecystectomy \\
\hline
\end{tabular}

began immediately after surgery but didn't resolve. The patient had a normal barium swallow and normal EGD. A gastric emptying study showed delayed gastric emptying of liquids and almost no emptying of solids. Given her chronic gastroparesis and absence of these symptoms preoperatively, it is presumed that this is secondary to a vagal nerve injury.

During esophagomyotomy one patient had an enterotomy at the base of the gastric myotomy. This was seen intraoperatively, repaired and then covered with a Dor fundoplication. On postoperative day (POD) 1 the patient had a gastrograffin swallow which was negative. The remainder of our complications are presented in Table 4.

\subsection{Conversions}

Nissen fundoplication conversions to laparoscopy:

Robot malfunction: upon mobilizing the hernia sac, one of the robot's arms repeatedly malfunctioned and auto defaulted several times. The robot was disengaged and the remainder of the mobilization of the hernia sac, paraesophageal hernia closure, and fundoplication were performed laparoscopically.

Collis gastroplasty: in three patients, the gastroesophageal junction was at or within the hiatal orifice, necessitating a collis gastroplasty to allow space for the fundoplication. Conversion to laparoscopy was done to perform the collis gastroplasty using a stapling device to excise a portion of the fundus, suture the diaphragmatic defect, and create the fundoplication.

Hiatal hernia repair conversion to laparoscopy:

Robot malfunction: one robotic arm repeatedly reset itself due to a mechanical malfunction of the gimble. We were unable to continue robotically, the robot was disengaged and the procedure was performed laparoscopically.

\subsection{Comparison of Results to Published Literature}

\section{Complications}

The published literature reports a perioperative complication average of 7.3\% (0 - 24) for robotic Nissen fundoplication patients [4-13], 6.4\% (3 - 13) for esophagomyotomy patients [16-19], and 15\% (0 - 31) for hiatal hernia repair patients [19-21]. Our series had similar results with $7 \%$ of Nissen fundoplication patients, $15 \%$ of esophagomyotomy patients, and $11 \%$ of hiatal hernia repair patients experiencing perioperative complications. The majority of our complications were general perioperative complications in nature and not specifically related to the foregut location of the procedure or the robotic device itself (Table 2).

In the robotic Nissen fundoplication literature, Hartmann et al. reported 118 cases and denied any perioperative complications [5]. Talamini et al. [11] looked at 25 cases and reported two perioperative complications (8\%). The first was a misshaped fundoplication requiring reoperation to correct the problem and the second was a gastric leak that occurred where a short gastric vessel was ligated through excessive periomental fat. Draaisma et al. [10] looked at 25 cases and reported six perioperative complications (24\%). These included liver capsule tears in 2 patients, minor bleeding in 2 patients, dysphagia post operatively due to the fundoplication being too tight requiring re-operation, and an incisional hernia 3 months post-operatively requiring repair.

Melvin et al. [17] looked at 104 patients who underwent robotic esophagomyotomy and reported six complications (5.8\%). These included gastrointestinal bleeding, nausea, hypertension, aspiration, and a colon injury secondary to initial trocar insertion. No foregut mucosal 
Table 4. Robotic foregut publications.

\begin{tabular}{|c|c|c|c|c|c|c|c|c|}
\hline Author & Year & $\mathrm{n}$ & $\begin{array}{l}\text { Total Case Time } \\
\text { min (range) }\end{array}$ & $\begin{array}{c}\text { Complications } \\
\%\end{array}$ & Conversion n (\%) & $\begin{array}{l}\text { Conversion to } \\
\text { Laparotomy n }\end{array}$ & $\begin{array}{l}\text { Conversion to } \\
\text { Laparoscopy n }\end{array}$ & $\begin{array}{c}\text { LOS } \\
\text { days (range) }\end{array}$ \\
\hline \multicolumn{9}{|c|}{ Nissen Fundoplication Series } \\
\hline Crawford & 2009 & 71 & $175(102-321)$ & 7 & $4(5.6)$ & 0 & 4 & $1.7(0-9)$ \\
\hline Hartmann [4] & 2009 & 18 & 207 & 6 & 0 & 0 & 0 & NA \\
\hline Hartmann [5] & 2008 & 118 & 105 & 0 & $6(5)$ & 0 & 6 & $4.2(2-10)$ \\
\hline Heemskerk [6] & 2007 & 11 & 176 & 9 & 0 & 0 & 0 & NA \\
\hline Morino [7] & 2006 & 25 & $78(48-104)$ & 0 & $1(4)$ & 0 & 1 & $3(2-7)$ \\
\hline Nakadi [8] & 2006 & 9 & 137 & 11 & $1(9)$ & 0 & 1 & 4.4 \\
\hline Muller-Stich [9] & 2006 & 20 & $65(40-130)$ & 5 & 0 & 0 & 0 & 2.9 \\
\hline Draaisma [10] & 2006 & 25 & $120(80-180)$ & 24 & 0 & 0 & 0 & $3(2-6)$ \\
\hline Talamini [11] & 2002 & 25 & 190 & 8 & $2(8)$ & 0 & 2 & NA \\
\hline Melvin [12] & 2002 & 20 & $141(88-271)$ & 0 & NA & NA & NA & $1.1(1-2)$ \\
\hline Cadiere [13] & 2001 & 10 & $76(59-130)$ & 10 & 0 & 0 & 0 & $1(1-4)$ \\
\hline \multicolumn{9}{|c|}{ Esophagomyotomy Series } \\
\hline Crawford & 2009 & 26 & $178(105-262)$ & 15.4 & 0 & 0 & 0 & $1.2(0-6)$ \\
\hline Huffmann [14] & 2007 & 24 & 355 (332 - 378) & NA & NA & NA & NA & 2.8 \\
\hline Iqbal [15] & 2006 & 19 & NA & NA & NA & NA & NA & 3.1 \\
\hline Galvani [16] & 2006 & 54 & $162(62-210)$ & 3.7 & 0 & 0 & 0 & $1.5(0.8-4)$ \\
\hline Melvin [17] & 2005 & 104 & 141 & 5.8 & $2(2)$ & 1 & 1 & 1.5 \\
\hline Horgan [18] & 2005 & 59 & $141(92-190)$ & 3.4 & 0 & 0 & 0 & $1.5(0.8-4)$ \\
\hline Ruurda [19] & 2005 & 24 & $95(75-170)$ & 12.5 & 3 (12.5) & 2 & 1 & $4(2-4)$ \\
\hline Talamani [11] & 2002 & 5 & 218 & NA & NA & NA & NA & NA \\
\hline \multicolumn{9}{|c|}{ Hiatal Hernia Repair Series } \\
\hline Crawford & 2009 & 35 & 177 & 11.1 & $1(3.7)$ & 0 & 1 & $2.3(1-14)$ \\
\hline Braumann [20] & 2008 & 14 & $134(80-240)$ & 0 & 0 & 0 & 0 & $5(1-14)$ \\
\hline Draaisma [21] & 2006 & 38 & $127(80-210)$ & 13.2 & $4(10.5)$ & 4 & 0 & $5(2-39)$ \\
\hline Ruurda [19] & 2005 & 32 & $130(85-210)$ & 31.3 & $3(9.4)$ & 3 & 0 & $5(2-39)$ \\
\hline
\end{tabular}

perforation was reported. Horgan et al. [18] looked at 59 robotic esophagomyotomy patients and reported 2 complications (3.4\%). These included an incisional hernia on POD 4 and a delayed colon perforation requiring colectomy. No foregut mucosal perforations were reported. Ruurda et al. [19] reported two mucosal perforations during esophagomotomy. Both occurred after finding inadequate pressure falls with intraoperative manometry leading to continued muscular dissection. Intraoperative manometry has been abandoned by this author because of these complications. We had a slightly higher compli- cation rate in our esophagomyotomy group compared to the published literature. Our series had one mucosal breach as described above. Only four $[16,17,19]$ out of the seven published robotic esophagomyotomy papers discuss complications, and therefore, as with operative times, a detailed comparison is challenging (Table 4).

Draaisma et al. [21] looked at 38 patients who underwent robotic hiatal hernia repairs and reported 5 perioperative complications (13.2\%). These included strangulated incisional hernia, esophageal leak, recurrence due to suture rupture, cardiac failure, and pneumonia. They 
also reported 4 patients (13\%) with long term complications which included recurrence of the hiatal hernia, partial herniation of the Toupet fundoplication, and a diaphragm tear with herniation. This is a total complication rate of $23.6 \%$. The breakdown of our own complication rate of 11\% (3/27) is shown in Table 2.

\subsection{Conversions}

The published literature reports a conversion rate average of 2.9\% (0 - 9) for Nissen fundoplication patients [4-13], $3.4 \%$ ( 0 - 12.5) for esophagomyotomy patients [16,17, 19], and $6.6 \%(0-10.5)$ for hiatal hernia repair patients [19-21]. Of these, all of the Nissen fundoplication conversions were to laparoscopy, while all of the hiatal hernia conversions were to laparotomy. The esophagomyotomy conversions were to both laparoscopy and to laparotomy (Table 4). Our series had similar results with a conversion rate of $5.6 \%$ of Nissen fundoplication patients, $0 \%$ of esophagomyotomy patients, and $3.7 \%$ of hiatal hernia repair patients (Table 1).

Hartmann et al. [5] reported a 5\% (6/118) conversion rate to laparoscopy during robotic fundoplication. Conversions were secondary to severe adhesions in 4 cases, failure of trocar position in 1 case, and system malfunction in 1 case. There were no conversions to laparotomy. Talamini et al. [11] reported an 8\% (2/25) conversion rate to laparoscopy and no conversions to laparotomy during their Nissen fundoplications. One conversion was secondary to a system failure and the second due to a system positioning problem.

Melvin et al. [17] reported a 2\% (2/104) total conversion rate during robotic esophagomyotomy. One case was converted to laparoscopy secondary to a system failure and the second was converted to laparotomy secondary to bleeding. Ruurda et al. [19] reported a $12.5 \%$ (3/24) conversion rate during robotic esophagomyotomy. Two conversions were to laparotomy due to mucosal injuries. The third was not expanded upon. Galvani et al. [16] and Horgan et al. [18] reported no conversions to laparoscopy or laparotomy during robotic esophagomyotomies when reporting 54 and 59 cases respectively.

Draaisma et al. [21] reported a conversion rate of $10.5 \%$ (4/38) during robotic hiatal hernia repairs. All four conversions were to laparotomy due to inadequate exposure. Ruurda et al. [19] reported a conversion rate of 9.4\% (3/32) during robotic hiatal hernia repairs. Again all conversions were to laparotomy, but the author does not discuss the reasons for the conversions.

As discussed earlier, all of our conversions were to laparoscopy. Two were from robotic malfunctions and four were to allow us to use staplers to perform a wedge resection Collis gastroplasty. The senior author (DLC) already had a large experience in laparoscopic foregut surgery which contributed to our low conversion rate.

\subsection{Total Case Time}

It is very difficult to directly compare the procedure times amongst the published series, since the authors/ surgeons record the operative times differently. Most studies recorded the time from skin incision to skin closure; this is the same as our TCT.

The mean TCT in the robotic Nissen fundoplication literature ranges from 65 - 207 minutes [4-13]. Our mean TCT was 173 minutes, which is within that range but on the higher end. Three studies had much shorter TCTs compared to the rest of the fundoplication series. Muller-Stich et al. [9] reported 20 cases with a mean TCT of 65 minutes. In this study the Nissen fundoplication was performed without dividing any of the short gastric vessels, which undoubtedly saved time. Muller-Stich et al. [9] compared robotic assisted versus laparoscopic Nissen fundoplications and thus far is the only study to have significantly shorter case times with the robotic group than the laparoscopic group. This was attributed to a highly experienced robotic surgeon and a very specialized, experienced, and consistent surgical team. Cadiere et al. [13] reported 10 cases with a mean TCT of 76 minutes. In this study the mobilization of greater curvature of the stomach was done laparoscopically and not robotically. The study does not address the reasoning for this but this could account for a shorter TCT. Morino et al. [7] reported 25 cases with a mean TCT of 78 minutes. Although no considerable operative differences were noted, any patient with a history of a previous upper abdominal surgery was excluded from the study. This excluded seven patients from the study (23\%). We did not exclude these patients in our study and therefore the few patients in our series that underwent extensive lysis of adhesions had longer operative times and contributed to our higher mean TCT. Hartmann et al. [5] looked at 118 patients and reported a median TCT of 105 minutes. They did not report a mean TCT, so direct comparison is not possible. This series performed a variety of fundoplications for the treatment of gastroesophageal reflux disease including Dorr (111 patients), Nissen (16 patients), and Toupet (1) fundoplications. The majority of the patients had a $180^{\circ}$ anterior partial fundoplication. The series doesn't compare the time it took to perform the Nissen versus the Dorr fundoplication.

The mean TCT in the robotic esophagomyotomy series ranged from 95 - 355 minutes [11,14,16-19]. Huffman et al. [14] reported the longest operative time with an average of 355 minutes, however they measured their case time from induction of anesthesia to extubation as opposed to skin incision to skin closure. Therefore we cannot compare our TCT with this study. Excluding this 
study, our mean TCT was longer than most of the others in the literature. In our series, seventy percent of the patients had a toupet fundoplication (partial $270^{\circ}$ posterior wrap) in addition to the esophagomyotomy. In other studies $[14,16,18]$, the patients had a dorr fundoplication (partial $180^{\circ}$ anterior wrap) in addition to the esophagomyotomy. Compared to the dorr fundoplication, the toupet fundoplication is technically more difficult and time consuming, requiring mobilization of the entire gastroesophageal junction circumferentially. This fact along with our significant resident involvement may contribute to our longer operative times.

The mean TCT in the robotic hiatal hernia repair series ranged from 127 - 134 minutes [19-21]. Our mean TCT was 169 minutes, again longer than the literature. Fiftytwo percent of patients in our series had an anterior gastropexy, lengthening the TCT (Table 3). None of the series in the literature discuss performing an anterior gastropexy with the hiatal hernia repair. In addition, on many occasions in 2009 and continuing still, we placed human allograft over the primary closure of the defect. We have recently begun recording how long this takes over and above the primary closure for study purposes. Several allograft placement cases were included in this series. Diaphragmatic closure of these large defects is usually when the senior author is most likely to let a junior resident sit at the console to experience suturing.

Thirty-five percent (44/124) of our patients had additional procedures performed at the time of their operations (Table 3). The published robotic foregut literature does not discuss performing any additional procedures during cases. The robotic Nissen fundoplication literature discusses performing hiatal hernia repairs in the majority of patient with gastroeophageal reflux disease. This is consistent with our series, with $98.6 \%$ (70/71) having hiatal hernia repairs performed with the Nissen fundoplication. One patient did not have a hiatal hernia repair due to the patient's very narrow crus around the esophagus. Twenty-five (35\%) of our Nissen fundoplication patients had additional procedures performed besides the hiatal hernia repair. Of the esophagomyotomy patients, most studies performed a partial fundoplication. This is consistent with our series. However, $7.6 \%$ of our patients had additional procedures at the time of surgery. Sixty-three percent (17/27) of our patients that underwent hiatal hernia repairs had additional procedures at the time of surgery. All of these additional procedures (Table 3) added to the length of our mean TCT. These are not seen in other robotic foregut series and could explain why our mean TCT is longer than other studies in all three groups.

\subsection{Length of Stay}

The published literature reports an average LOS of 3.4
(1 - 7.2) days for robotic Nissen fundoplication patients [4-13], 2.4 (1.5 - 4) days for esophagomyotomy patients [8,14-18], and 5 days for hiatal hernia repair patients [19-21]. Our series had a shorter LOS for all groups with a mean LOS of 1.7 days for all three procedures combined, 1.7 days for the Nissen fundoplication group, 1.2 days for the esophagomyotomy group, and 2.3 days for the hiatal hernia repair group.

Of the robotic Nissen fundoplication studies, Hartmann et al. [5] had an average LOS of 4.2 (2 - 10) days for 118 patients, Draaisma et al. [10] had an average LOS of 3 (2-6) days for 25 patients, and Melvin et al. [12] had an average LOS of $1.1(1-2)$ days for 20 patients. Of the robotic esophagomyotomy studies, Melvin et al. [17] had an average LOS of 1.5 days for 104 patients, Horgan et al. [18] had an average LOS of 1.5 (0.8 - 4) days for 59 patients, and Ruurda et al. [19] had an average LOS of $4(2-6)$ days for 24 patients. Of the robotic hiatal hernia repair studies, Braumann et al. [20], Ruurda et al. [19], and Draaisma et al. [21] all had an average LOS of 5 days for 14, 32, and 38 patients respectively (Table 4).

Our hiatal hernia average LOS was 2.3 days, while this is shorter than the published literature, it was longer than the LOS compared to our Nissen fundoplication and esophagomyotomy groups. This was likely due to the older age of this patient group with a mean age of 71 years compared to 50 years in the other two groups. Therefore due to the older age, the hiatal hernia group had more comorbidities compared with the other two groups, contributing to longer hospital stays.

Although our mean LOS was shorter for all three groups compared to the published literature, a portion of the literature is from European countries. Differences in health care coverage in the United States compared with other countries (esp. Europe) as well as societal expectations of convalescence may contribute to the differences in length of stay. Most of the literature for both the robotic Nissen fundoplication and hiatal hernia groups comes from European countries.

\subsection{Resident Participation}

Residents participated in $68.5 \%$ of all robotic foregut cases. Resident involvement in the cases begins during the intern year and participation increases throughout residency. Overall resident participation increased each year throughout the study with residents involved in 52\% of cases in the 1st four years of the series and $86 \%$ of cases during the 2nd four years. In 2002, there were no residents involved in the robotic foregut cases. Resident involvement increased dramatically in subsequent years with involvement in $81 \%$ of cases in 2009. Figure 2 shows the percentage of resident involvement in cases 


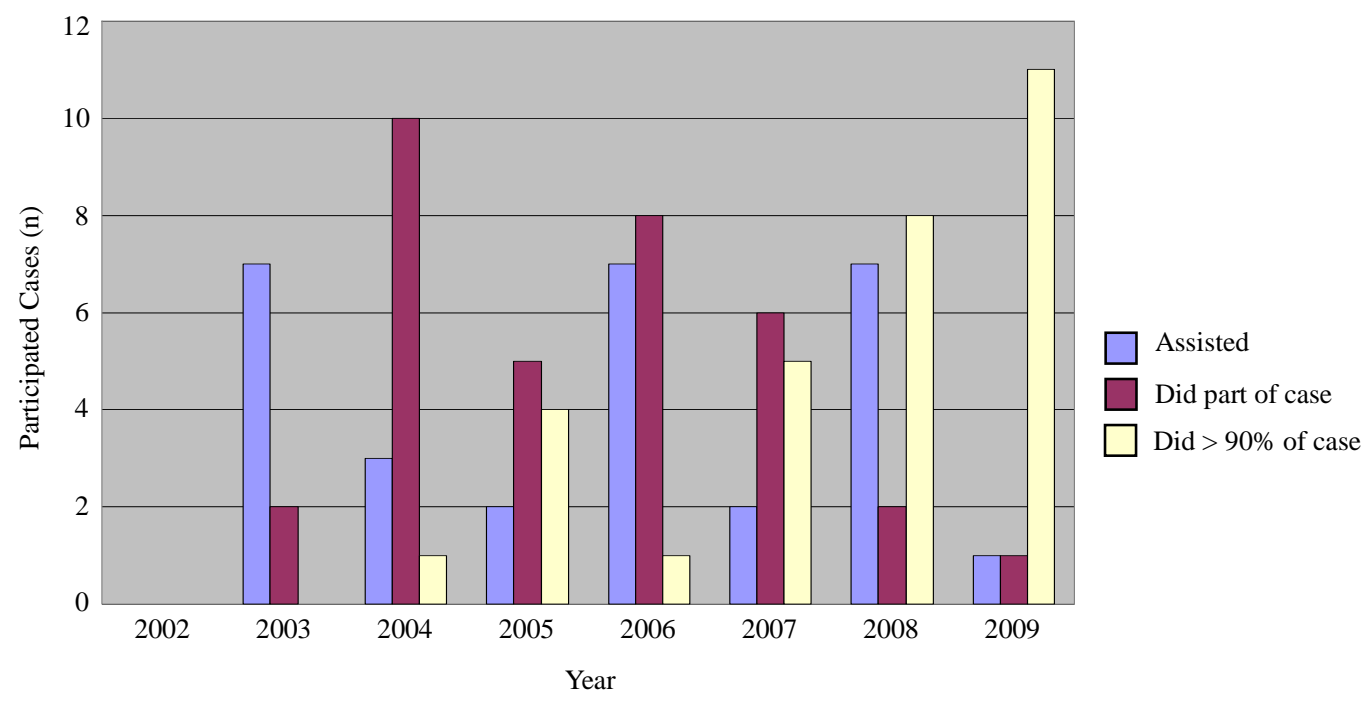

Figure 2. Resident participation 2002-2009.

each year from 2002-2009.

For a more meaningful demonstration of the increase in resident participation, we included cases that were performed through the end of December 2009 after the study end date (June 2009). During the 1st few years of the study, the residents mainly assisted the senior author. By the last few years of the study, the majority of the cases had residents performing greater than 90 percent of each case (see the white bars in Figure 2). Early junior resident participation in foregut cases is possible because of the senior author's large laparoscopic experience in the foregut. Second year residents frequently close the diaphragm after mobilization is completed. Beginning in the 3rd year, residents start performing more than $90 \%$ of the cases. The 4th year involvement decreases due to the reduced access this year has to the device having two thirds of their year made up of trauma and night float, both services where the robot is not used. Fifth year residents do more than $90 \%$ of the cases the majority of the time, while the senior author remains as the instructor and assistance at the operative table. The incorporation of robotic training in the surgical curriculum can easily be done and serves as a great teaching tool that exposes the residents to new technology.

\section{Conclusion}

Robotic-assisted foregut surgery is safe and effective. This series compares favorably with other robotic studies in length of hospital stay, total case time, and rates of complication and conversion. Resident participation was significant and is expected to continue to increase. Foregut surgery is an excellent training ground for residents with its narrow operative field and diversity of skills required to complete the procedures.

\section{Disclosures}

David Crawford, Franziska Huettner, Robin Alley, Jamie Doubet, Mike Ryan, and Danuta Dynda have no conflicts of interest or financial ties to disclose. We have full control of all primary data and we agree to allow the journal to review our data if requested.

\section{REFERENCES}

[1] S. Horgan and D. Vanuno, "Technical Report Robots in Laparoscopic Surgery,” Journal of Laparoendoscopic \& Advanced Surgical Techniques, Vol. 11, No. 6, 2001, pp. 415-419. doi:10.1089/10926420152761950

[2] G. B. Cadiere, J. Himpens, M. Vertruyen, J. Bruyns and G. Fourtanier, "Nissen Fundoplication Done by Remotely Controlled Robotic Technique," Annales de Chirurgie, Vol. 53, No. 2, 1999, pp. 137-141.

[3] W. S. Melvin, K. R. Krause, B. J. Needleman, R. K. Wolf, R. E. Michler and E. C. Ellison, "Computer Assisted 'Robotic’ Heller Myotomy: Initial Case Report,” Journal of Laparoendoscopic \& Advanced Surgical Techniques, Vol. 11, No. 4, 2001, pp. 251-253. doi:10.1089/109264201750539790

[4] J. Hartmann, C. Menenakos, J. Ordemann, W. Raue and C. Braumann, "Long-Term Results of Quality of Life after Standard Laparoscopic vs Robot-Assisted Laparoscopic Fundoplications for Gastro-Oesophageal Reflux Disease. A Comparative Trial," International Journal of Medical Robotics and Computer Assisted Surgery, Vol. 5, No. 1, 2009, pp. 32-37. doi:10.1002/rcs.228

[5] J. Hartmann, C. A. Jacobi, C. Menenakos, M. Ismail and C. Braumann, "Surgical Treatment of Gastroesophageal Reflux Disease and Upsidedown Stomach Using the da Vinci Robotic System. A Prospective Study,” Journal of Gastrointestinal Surgery, Vol. 12, No. 3, 2008, pp. 504509. doi:10.1007/s11605-007-0400-z

[6] J. Heemskerk, W. G. Van Gemert, J. W. Greve and N. D. 
Bouvey, "Robot-Assisted versus Conventional Laparoscopic Nissen Fundoplication: A Comparative Retrospective Study on Costs and Time Consumption,” Surgical Laparoscopy Endoscopy Percutaneous Techniques, Vol. 17, No. 1, 2007, pp. 1-4. doi:10.1097/01.sle.0000213756.76761.b7

[7] M. Morino, L. Pellegrino, C. Giaccone and F. Rebecchi, "Randomized Clinical Trial of Robot-Assisted versus Laparoscopic Nissen Fundoplication," British Journal of Surgery, Vol. 93, No. 5, 2006, pp. 553-558. doi:10.1002/bjs. 5325

[8] E. L. Nakadi, C. Melot, J. Closset, V. DeMoor, K. Betroune, P. Feron, P. Lingier and M. Gelin, "Evaluation of da Vinci Nissen Fundoplication Clinical Results and Cost Minimization,” World Journal of Surgery, Vol. 30, No. 6, 2006, pp. 1050-1054. doi:10.1007/s00268-005-7950-6

[9] B. P. Muller-Stich, M. A. Reiter, M. N. Wente, V. V. Bintintan, J. Koninger, M. W. Buchler and C. N. Gutt, "Robot-Assisted versus Conventional Laparoscopic Fundoplication: Short-Term Outcome of a Pilot Randomized Controlled Trial,” Surgical Endoscopy, Vol. 21, No. 10, 2007, pp. 1800-1805. doi:10.1007/s00464-007-9268-y

[10] W. A. Draaisma, J. P. Ruurda, C. H. Schefer, R. K. J. Simmermacher, H. G. Gooszen, H. G. Rijnhart-De Jong, E. Buskens and I. A. M. J. Broeders, "Randomized Clinical Trial of Standard Laparoscopic versus Robot-Assisted Laparoscopic Nissen Fundoplication for Gastro-Oesophageal Reflux Disease,” British Journal of Surgery, Vol. 93, No. 11, 2006, pp. 1351-1359. doi:10.1002/bjs.5535

[11] M. Talamini, K. Campbell and C. Stanfield, "Robotic Gastrointestinal Surgery: Early Experience and System Description,” Journal of Laparoendoscopic \& Advanced Surgical Techniques, Vol. 12, No. 4, 2002, pp. 225-232. doi:10.1089/109264202760267970

[12] W. S. Melvin, B. J. Needleman, K. R. Krause, C. Schneider and E. C. Ellison, "Computer-Enhanced vs Standard Laparoscopic Antireflux Surgery,” Journal of Gastrointestinal Surgery, Vol. 6, No. 1, 2002, pp.11-16. doi:10.1016/S1091-255X(01)00032-4

[13] G. B. Cadiere, J. Himpens, M. Vertruyen, J. Bruyns, O. Germay, G. Leman and R. Izizaw, "Evaluation of Telesurgical (Robotic) Nissen Fundoplication,” Surgical Endoscopy, Vol. 15, No. 9, 2001, pp. 918-923. doi:10.1007/s004640000217

[14] L. C. Huffmann P. K. Pandalai, B. J. Boulton, L. James, S.
L. Starnes, M. F. Reed, J. A. Howington and M. S. Nussbaum, "Robotic Heller Myotomy: A Safe Operation with Higher Postoperative Quality-of-Life Indices,” Surgery, Vol. 142, No. 4, 2007, pp. 613-620. doi:10.1016/j.surg.2007.08.003

[15] A. Iqbal, M. Haider, K. Desai, N. Garg, J. Kavan, S. Mittal and C. J. Filipi, "Technique and Follow-Up of Minimally Invasive Heller Myotomy for Achalasia," Surg Endosc, Vol. 20, 2006, pp. 394-401. doi:10.1007/s00464-005-0069-X

[16] C. Galvani, M. V. Gorodner, F. Moser, M. Baptista, P. Donahue and S. Horgan, "Laparoscopic Heller Myotomy for Achalasia Facilitated by Robotic Assistance,” Surgical Endoscopy, Vol. 20, No. 7, 2006, pp. 1105-1112. doi:10.1007/s00464-005-0272-9

[17] W. S. Melvin, J. M. Dundon, M. Talamini and S. Horgan, "Computer-Enhanced Robotic Telesurgery Minimizes Esophageal Perforation during Heller Myotomy,” Surgery, Vol. 138, No. 4, 2005, pp. 553-559. doi:10.1016/j.surg.2005.07.025

[18] S. Horgan, C. Galvani, M. V. Gorodner, P. Omelanczuck, F. Elli, F. Moser, L. Durand, M. Carcoche, J. Nefa, S. Bustos, P. Donahue and P. Ferraina, "Robotic-Assisted Heller Myotomy versus Laparoscopic Heller Myotomy for the Treatment of Esophageal Achalasia: Multicenter Study," Journal of Gastrointestinal Surgery, Vol. 9, No. 8, 2005, pp. 1020-1030. doi:10.1016/j.gassur.2005.06.026

[19] J. P. Ruurda, W. A. Draaisma, R. van Hillegersberg, I. H. M. B. Rinkes, H. G. Gooszen, L. W. M. Janssen, R. K. J. Simmermacher and I. A. M. J. Broeders, "Robot-Assisted Endoscopic Surgery: A Four-Year Single-Center Experience,” Digestive Surgery, Vol. 22, No. 5, 2005, pp. 313320. doi:10.1159/000088628

[20] C. Braumann, C. A. Jacobi, C. Menenakos, M. Ismail, J. C. Rueckert and J. M. Mueller, "Robotic-Assisted Laparoscopic and Thoracoscopic Surgery with the da Vinci: A 4-Year Experience in a Single Institution,” Surgical Laparoscopy Endoscopy Percutaneous Techniques, Vol. 18, No. 3, 2008, pp. 260-266. doi:10.1097/SLE.0b013e31816f85e5

[21] W. A. Draaisma, H. G. Gooszen and I. A. M. J. Broeders, "Mid-Term Results of Robot-Assisted Laparoscopic Repair of Large Hiatal Hernias: A Symptomatic and Radiologic Follow-Study," Surgical Laparoscopy Endoscopy Percutaneous Techniques, Vol. 16, No. 4, 2006, p. 300. doi:10.1097/00129689-200608000-00070 